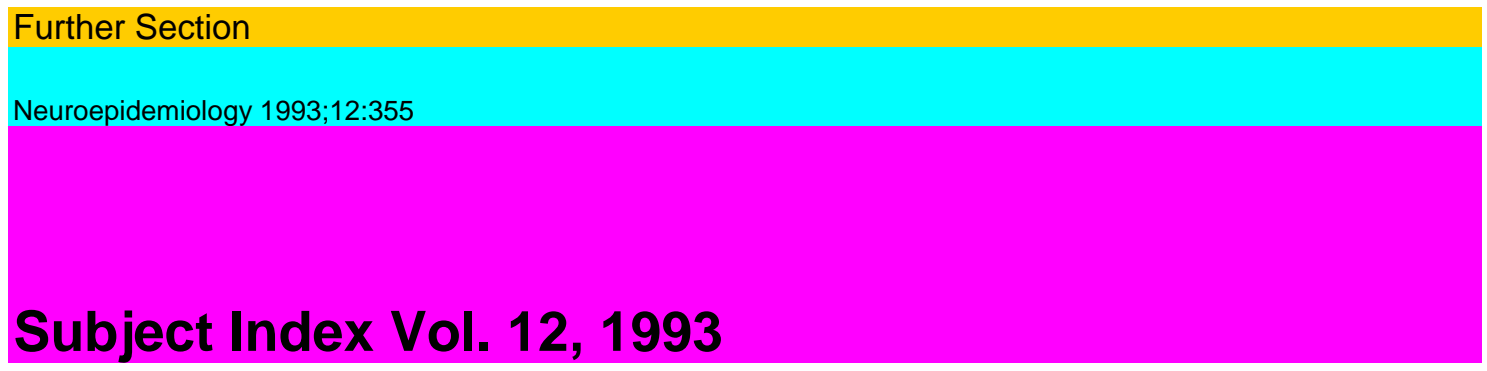

Acupuncture 106

Adrenoleukodystrophy 229

Adventist 28

Aged 96

Alcohol 43,249

Algorithm 51

Alzheimer's disease 28, 219,336,345

Amyotrophic lateral sclerosis 114,219,262

Anticoagulation therapy 280

Arab population 164

Aspirin 280

Becker dystrophy 326 Blood transfusion 158 Body mass index 43 Bovines 15 Brain tumors 270

Cardiac arrhythmia 241 Case-control study 6,

61,70 Cerebral isch(a)emia 70,

141 Cerebrovascular

disease(s) 37,43,280 - disorders 82 Cigarette smoking 141 Classification 51 Clinical trial 106 Cluster analysis 6

Community survey 164 Cortical ischaemia 141 Cow milk 15 Cycad 307 Cytochrome P-450 114

Dementia 219 Depression 179 Diabetes mellitus 241

Diagnosis 51 Diet 28

Double censoring 300 Duchenne dystrophy

326 Dystrophin 326

Environmental factors 209

- neurotoxins 114Epidemiology 15,37,

43,82,88,148,195, 229,249,313,326, 336, 345

-, hospital-based 257

Epilepsy 285

Etiology 6, 234, 257

Evaluation 51

Facial nerve diseases 313

- paralysis 313Farm animals 15 Feasibility study 106

Gangliosides 251 Geography 1 Guillain-Barr6 syndrome 251

Headache 179 Heterogeneity 6 Hispanic Americans 313 House pets 15 Hypertension 241

Incidence 88,195,270 Infections 61 Ischemic stroke 106 Italy 82

Kii Peninsula 307

Latent period 300

Migraine 51,179,249,

285 - personality 179 Mononeuropathy 257 Mortality 148,219 Motor neuron disease

307, 331 Multiple sclerosis 1,6,

15,61,158,234,300 Muscular dystrophy 262 Myasthenia gravis 262 Myocardial infarction

241 Myositis 262 Myotonia 262

Neuroepidemiology 285 Neurological disorders

164 Neuropathy 251,262 -, generalized 257 Non parametric model 
300 Nonvalvular atrial fibrillation 280 Nutritional factors 234

\section{Olfaction 114}

Parkinsonism 114,336,

345 Parkinson's disease 195,

209,219,285,336,

345 Peroxisomal disorder

229 Physical activity 43 Polyneuritis occurrence

96 Polyneuropathy 257

Prevalence 1,164,195,

262, 285 Primary degenerative

dementia 336, 345

Retroviruses 158

Risk factor(s) 37, 70, 96,

141,209,241,280,

313

Saudi Arabia 164 Senile dementia 28 Serotonin 249 Sleep latency 249 Smoking 43 Spain 251 Spinal cord injury 88 - muscular atrophy331

Stroke 141, 148,285

- recurrence 241Substance abuse 6Survival 270Susceptibility 1

Tension-type headache

179 Texas 313 Transient ischemic

attack 241 Trends 148

Vegetarian 28 Viral infection 209

Warfarin 280

X. X-linked disorder 229 